\title{
Tumor Microenvironment Varies under Different TCM ZHENG Models and Correlates with Treatment Response to Herbal Medicine
}

\author{
Zhen Chen, ${ }^{1,2}$ Lian-Yu Chen, ${ }^{1,2}$ Peng Wang, ${ }^{1,2}$ Hai-Yan Dai, ${ }^{1,2}$ Song Gao, ${ }^{1,2}$ and Kun Wang1,2 \\ ${ }^{1}$ Department of Integrative Oncology, Fudan University Shanghai Cancer Center, Shanghai 200032, China \\ ${ }^{2}$ Department of Oncology, Shanghai Medical College, Fudan University, Shanghai 200032, China
}

Correspondence should be addressed to Peng Wang, wangp413@yahoo.com.cn

Received 11 January 2012; Accepted 1 April 2012

Academic Editor: Shi-Bing Su

Copyright ( $) 2012$ Zhen Chen et al. This is an open access article distributed under the Creative Commons Attribution License, which permits unrestricted use, distribution, and reproduction in any medium, provided the original work is properly cited.

\begin{abstract}
In traditional Chinese medicine (TCM), diagnosis of pathology and choice of treatment prescriptions are based on a method of differentiation of signs and symptoms known as syndrome differentiation or ZHENG. The cornerstone of TCM, ZHENG, relies on the gathering of clinical information through inspection, auscultation and olfaction, inquiry, and palpation. However, the biomolecular basis of the ZHENG remains unclear. In this study, we established mouse xenograft pancreatic cancer models with Shi-Re (Dampness-Heat), $\mathrm{Pi}-\mathrm{Xu}$ (Spleen-Deficiency), or Xue-Yu (Blood-Stasis) ZHENG, which are regarded as the three major ZHENGs in pancreatic cancer. We found that tumors of the different ZHENG models exhibited significantly altered cancerassociated fibroblast (CAF) proliferative activity and tumor-associated macrophage (TAM) infiltration, which led to altered levels of CAF- and TAM-derived secreted cytokines such as SDF-1 and CCL5. The ZHENG model type also significantly influenced tumor growth, and administration of herbal medicine to the ZHENG model modified the tumor microenvironment. Therefore, this study partially unveiled the molecular basis of TCM ZHENG in pancreatic cancer.
\end{abstract}

\section{Introduction}

Traditional Chinese medicine (TCM) has a history of over 3000 years. A holistic form of medicine, TCM, emphasizes bringing the patient's body, mind, and spirit into harmony. The theory and application of TCM are one of constant summarizing, inducing, and refining of the experiences accumulated in preventing and treating diseases in daily life and medical practice.

TCM rests squarely on ZHENG (syndrome) differentiation, a process of analyzing data collected through four combined diagnostic methods: inspection, auscultation and olfaction, inquiry, and palpation. All diagnostic and therapeutic methods in TCM are based on the differentiation of ZHENG. In modern times, TCM has become popular worldwide because of its reliable therapeutic efficacy [1]. However, diagnosis in TCM depends on the intuition and experience of the physician grounded in TCM theory, and this method seems to lack objectivity, accuracy, and reproducibility in the face of biomolecular science and Western-based medicine.
Furthermore, the concept of $Z H E N G$ is often misinterpreted and unclear. For all these reasons, researchers from China and elsewhere have begun to investigate the ZHENG of TCM for a molecular foundation.

Tumors are now recognized as structures of multiple cell types, comparable to organs in complexity, which during tumorigenesis recruit the involvement of surrounding normal cells to construct and interact within a tumor microenvironment [2]. Continuous paracrine signaling with feedback within this microenvironment eventually leads to the end stages of cancer progression [3]. As cancer is no longer considered a discrete entity defined only by the traits of cancer cells within the tumor but may eventually involve the entire organism, TCM offers a holistic approach whose goal is regulating the integrity of all body functions as well as the interaction between the human and surrounding environment.

We have previously shown that the presence of ZHENG may influence tumor growth in pancreatic cancer. We also found that this effect might correlate with the CC chemokine 
( $\beta$-chemokine) family [4]. This finding suggests an involvement between ZHENG and the tumor microenvironment and deserves further research. Accordingly, in the present study we evaluated the tumor microenvironment under different ZHENG conditions, specifically noting changes in the proliferative activity of cancer-associated fibroblasts (CAF) and the infiltration of tumor-associated macrophages (TAM). We confirm here that characteristics of the tumor environment correlated with the ZHENG of TCM, and herbal treatments modified the tumor microenvironment.

\section{Materials and Methods}

2.1. Cell Lines and Mice. Samples of the pancreatic cancer cell line Panc02 were obtained from the Cancer Research and Development Center and grown in complete growth medium as recommended by the manufacturer. The cultured cells were maintained in a humidified $5 \% \mathrm{CO}_{2}$ atmosphere at $37^{\circ} \mathrm{C}$. All cells were regularly authenticated by observing cell morphology and tested for the absence of mycoplasma contamination using a MycoAlert Mycoplasma Detection kit (MycoAlert, Lonza, Rockland, ME, USA).

Male C57 mice, 4- to 6-week old, were obtained from the Shanghai Institute of Materia Medica at the Chinese Academy of Sciences (Shanghai, China) and housed in laminar flow cabinets under specific pathogen-free conditions with food and water supplied ad libitum. All experiments on mice were conducted in accordance with the guidelines of the National Institutes of Health (NIH) for the Care and Use of Laboratory Animals. The Committee for the Use of Live Animals in Teaching and Research, Fudan University, Shanghai approved the study protocol.

2.2. Drugs and Reagents. Individual packets of herb powders for each herb were produced by Jiangyin Tianjiang Pharmaceutical. The final decoction of each prescription was prepared at the Department of Pharmacy, Fudan University Shanghai Cancer Center, Shanghai, China, by dissolving the herb powder into distilled water to the required concentration. The daily dosage of herb decoctions for the mice was calculated according to the following human-mouse transfer formula: $D b=D a \times(R b / R a) \times(W b / W a) 2 / 3$, where $D, R$, and $W$ represent dosage, shape coefficient, and body weight, respectively, and $a$ and $b$ represent human mouse, respectively.

Honey supplied by Guan Sheng Yuan International Trade (Shanghai) was adjusted to a concentration of $20 \%$ in water. Wine (er guo tou) obtained from Hongxing (Beijing) was diluted to $55 \%$ in water. Pork fat was donated by $\mathrm{Gu}$ Jianzhong, Chinese Academy of Sciences (Shanghai). The following antibodies were used: anti-vimentin, anti- $\alpha$ smooth muscle actin (SMA), anti-C-X-C chemokine receptor type 4 (CXCR4), and anti-C-C chemokine receptor type 5 (CCR5; all from Epitomics), and anticluster of differentiation 68 (CD68; Santa Cruz Biotechnology, Santa Cruz, CA).

2.3. Establishment of TCM ZHENG Model. We developed three types of TCM ZHENG models in respective mouse groups, namely, Shi-Re (Dampness-Heat), Pi-Xu (SpleenDeficiency), and Xue-Yu (Blood-Stasis). The Shi-Re and $P i-X u$ ZHENG models were established as we described previously [4]. Briefly, the Shi-Re condition was established by the wine and the pork fat combination (day 1 to day 7, $0.2 \mathrm{~mL}$ ), and the food and honey-water were provided. $\mathrm{Pi}-\mathrm{Xu}$ was developed by feeding the mice with a decoction of mirabilite and Chinese rhubarb, $0.2 \mathrm{~mL}$ for each mouse (day 1 to day 7). The Xue-Yu ZHENG was established by subcutaneous injection of $0.01 \%$ adrenaline $(0.13 \mathrm{mg} / \mathrm{kg})$ for each mouse (day 1 to day 7), as we described previously [5].

2.4. Subcutaneous Xenograft Tumor Model. Panc02 cells $(2 \times$ $10^{6}$ cells in $200 \mu \mathrm{L}$ ) were injected subcutaneously into the right axilla of each C57 mouse. The length and width of tumors (in millimeters) were measured weekly with calipers. Tumor volume was calculated by the formula $\left(a \times b^{2}\right) \times$ 0.5 , where $a$ and $b$ were the long and short dimensions, respectively. Mice were euthanized under anesthesia when tumors reached $1.5 \mathrm{~cm}$ in diameter. The tumors were then resected and weighed. Each group had $\geq 10$ mice.

2.5. Immunohistochemical Analysis. Specimens of tumor tissue were fixed in $10 \%$ formalin and embedded in paraffin wax. Unstained $3 \mu \mathrm{m}$ sections were then cut from paraffin blocks for immunohistochemical (IHC) analysis. The sections were stained with rabbit anti-vimentin $(1: 100)$, rabbit anti- $\alpha$-SMA $(1: 100)$, rabbit anti-CD68 $(1: 200)$, rabbit antiCXCR4 $(1: 200)$, and rabbit anti-CCR 5 at $4{ }^{\circ} \mathrm{C}$ overnight. The secondary antibody and avidin-biotin peroxidase complex method was used according to the standard protocols provided by the manufacturer (Vector Laboratories, CA, USA). An immunoglobulin-negative control was used to rule out nonspecific binding. Two independent assessors and one pathologist performed all procedures, all of whom were blinded to the model/treatment type for this series of specimens.

To quantitatively evaluate the CAF proliferative activity and TAM infiltration in each group, we calculated the ratio of the area positive for vimentin or CD68 staining to the total area in histological sections from ten fields under light microscopy (200x). The procedure for evaluation of CXCR4 and CCR5 expression followed that of our previous report [4].

2.6. Enzyme-Linked Immunosorbent (ELISA) Assay for Cytokine Release. The concentrations of SDF-1 and CCL5 in the tumor samples were determined using a sandwich ELISA kit (DuoSet; R\&D Systems, Minneapolis, MN) according to the protocol of the manufacturer. Briefly, frozen tumor tissue was homogenized in lysis buffer and thereafter centrifuged at $12,000 \mathrm{rpm}$ for 15 minutes at $4^{\circ} \mathrm{C} ; 50 \mu \mathrm{L}$ of the supernatant was used for ELISA. Concentrations of immunoreactive SDF-1 and CCL5 were expressed as $\mathrm{pg} / \mathrm{mL}$.

2.7. Statistical Analyses. The data are expressed as the mean \pm standard error (SE) of three or more independent experiments performed in triplicate. The statistical analyses 
were performed using analysis of variance (ANOVA) models and Student's $t$-tests. A $P$ value $<0.05$ was accepted as statistically significant.

\section{Results}

3.1. ZHENG Distribution in Pancreatic Cancer Patients. We firstly investigated the distribution of ZHENG conditions in populations of pancreatic cancer patients based on reports published from January 1, 1998 to December 31, 2008. Sixty-nine studies were identified by electronic and hand searches, among which 34 clinical articles were included for our study. Data on $Z H E N G$ distribution were extracted and analyzed. Twenty-seven $Z$ HENGs were identified. The three $Z$ HENGs in pancreatic cancer that were most reported were Dampness-Heat (in Chinese, Shi-Re; 33.9\% of studies), Spleen-Deficiency ( $\mathrm{Pi}-\mathrm{Xu} ; 29.10 \%)$, and Blood-Stasis (XueYu; 19.8\%; Figure 1).

\subsection{Alteration of Tumor Microenvironment under Different} ZHENG Conditions. The tumor microenvironment plays an important role in the development and progression of cancer $[6,7]$. Pancreatic carcinomas are surrounded by desmoplastic stroma consisting of fibroblasts, immune cells, endothelial cells, and pericytes [8]. We hypothesized that the tumor microenvironment would be altered under different ZHENG conditions. To verify this hypothesis, we first established 3 subcutaneous tumor models of pancreatic cancer in mice that exemplified the ZHENG conditions Shi-Re, $\mathrm{Pi}$ $X u$, and $X u e-Y u$, respectively. We sought to investigate the differences in the tumor microenvironment among these ZHENG models.

As it is recognized that in many tumors the stroma is characterized by an increase in fibroblast proliferation, we immunostained CAFs using the fibroblastic marker vimentin combined with the defined myofibroblast marker $\alpha$-smooth muscle actin ( $\alpha$-SMA) to investigate the proliferative activity of CAF [9]. We found that the number of both vimentin- and $\alpha$-SMA-positive cells was decreased in tumors from the Shi$R e$ and $P i-X u Z H E N G$ models of pancreatic cancer compared with the control tumor, while tumors from the $X u e-Y u$ model exhibited no changes in CAF activity (Figures 2(a) and 2(b)). This observation suggested that CAF proliferative activity in tumors was altered differently on the basis of $Z H E N G$ conditions.

It is accepted that, in general, cancer- and host-cellderived signals program TAMs to acquire an M2-like polarized and otherwise tumor-supportive phenotype [10]. In many cases increased numbers of TAMs are associated with a poorer prognosis $[11,12]$. Therefore, we evaluated tumors in the different ZHENG models for TAM infiltration by staining for CD68 (also known as macrosialin in mice), a glycoprotein expressed on macrophages. We found that, compared with the control group, the number of macrophages was dramatically less in the Shi-Re group, followed by lesser degrees of decrease in the $P i-X u$ groups (Figures 2(a) and 2(b)). This observation suggests relatedness between the inflammation characteristics of tumor microenvironments and the specific

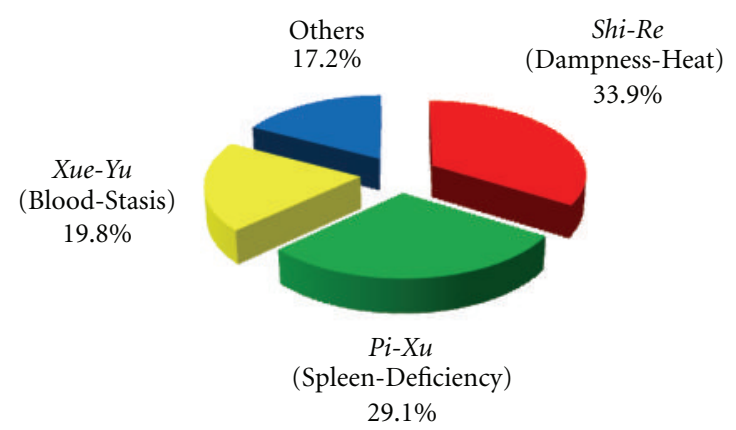

Figure 1: Percentages of pancreatic patients diagnosed with various TCM ZHENG conditions. Data on ZHENG distribution were extracted and analyzed from 34 clinical articles published between January 1, 1998 and December 31, 2008.

ZHENG conditions tested. Altogether, our study demonstrated a correlation between ZHENG conditions and the microenvironment of tumors in pancreatic cancer.

3.3. Correlation between Tumor Microenvironment and Growth under Different ZHENG Conditions. CAFs stimulate tumor cell proliferation and invasion through various growth factors, hormones, and cytokines [13]. SDF-1 is a CAF-derived chemokine that has been shown to directly boost the proliferation and invasion of pancreatic cancer cells [14]. Thus, we evaluated the levels of secreted SDF1 in tumors under different ZHENG conditions, and the expression of CXCR4, the SDF-1 cognate receptor, in tumor cells. The results of ELISA assays showed decreased levels of SDF-1 released in tumors in the Shi-Re and $\mathrm{Pi}-\mathrm{Xu}$ groups compared to the control mice. This was not observed in the $X u e-Y u$ tumors (Figure 3(a)). This result was consistent with the observation that the Shi-Re and $\mathrm{Pi}-\mathrm{Xu}$ tumors exhibited decreased CAF proliferative activity. However, there was no difference in CXCR4 expression among the ZHENG models and control tumor cells (Figures $3(\mathrm{~b})$ and $3(\mathrm{c})$ ).

Similarly, we wanted to verify whether the decreased TAM infiltration we observed above led to a reduction in the levels of the TAM-derived cytokine CCL5. We found that secreted CCL5 decreased dramatically in tumors under Shi$R e$ and $P i-X u Z H E N G$ s. This was also observed in the Xue$Y u$ tumors, although the difference was not significant $(P=$ 0.083 ) (Figure 3(a)). We also found that tumor cells from the Shi-Re, $P i-X u$, and $X u e-Y u$ models exhibited decreased CCR5 expression, especially for Shi-Re (Figures 3(b) and 3(c)).

After we confirmed that CAF-related SDF-1/CXCR4 and TAM-related CCL5/CCR5 expressions were changed under different $Z H E N G$ conditions, we next investigated an association between the altered tumor microenvironments and tumor growth. We found that altered tumor microenvironments were correlated with in vivo changes in tumor growth (Figure $3(\mathrm{~d})$ ). Taken together, these results suggest that tumors under different $Z H E N G$ conditions exhibited different tumor microenvironments, which may finally effect tumor growth. 

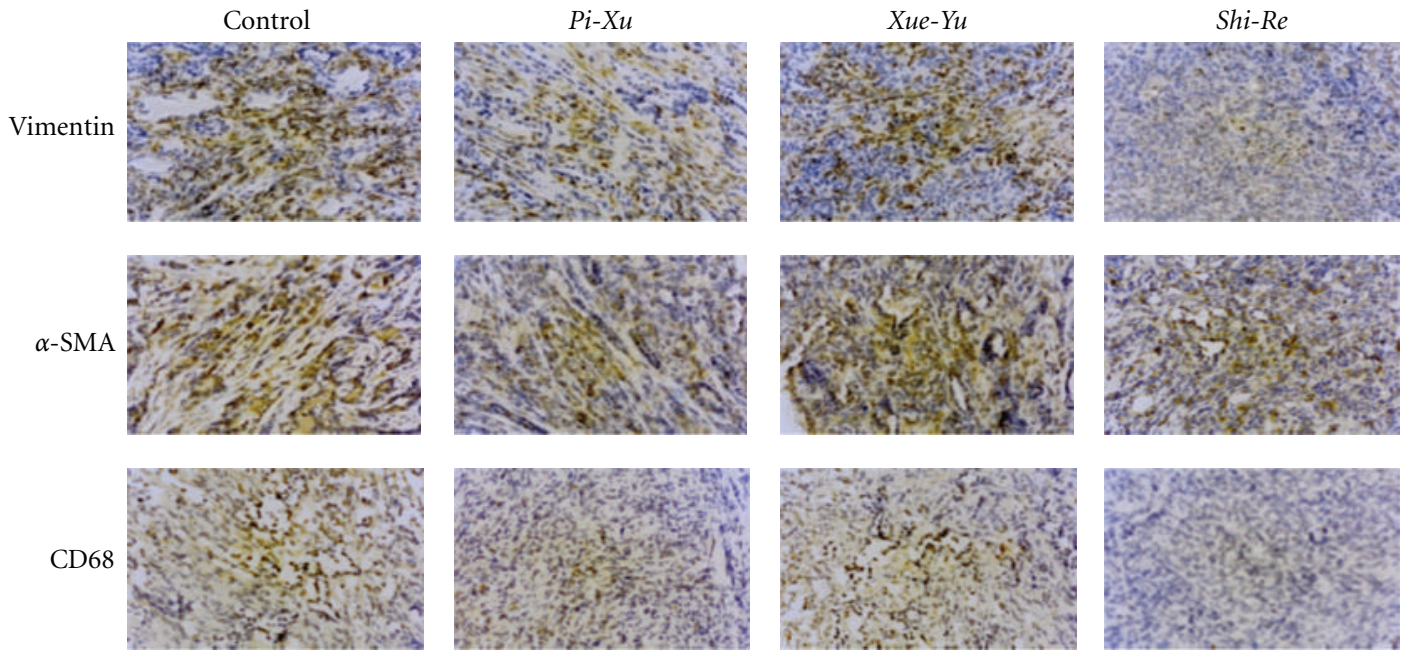

(a)
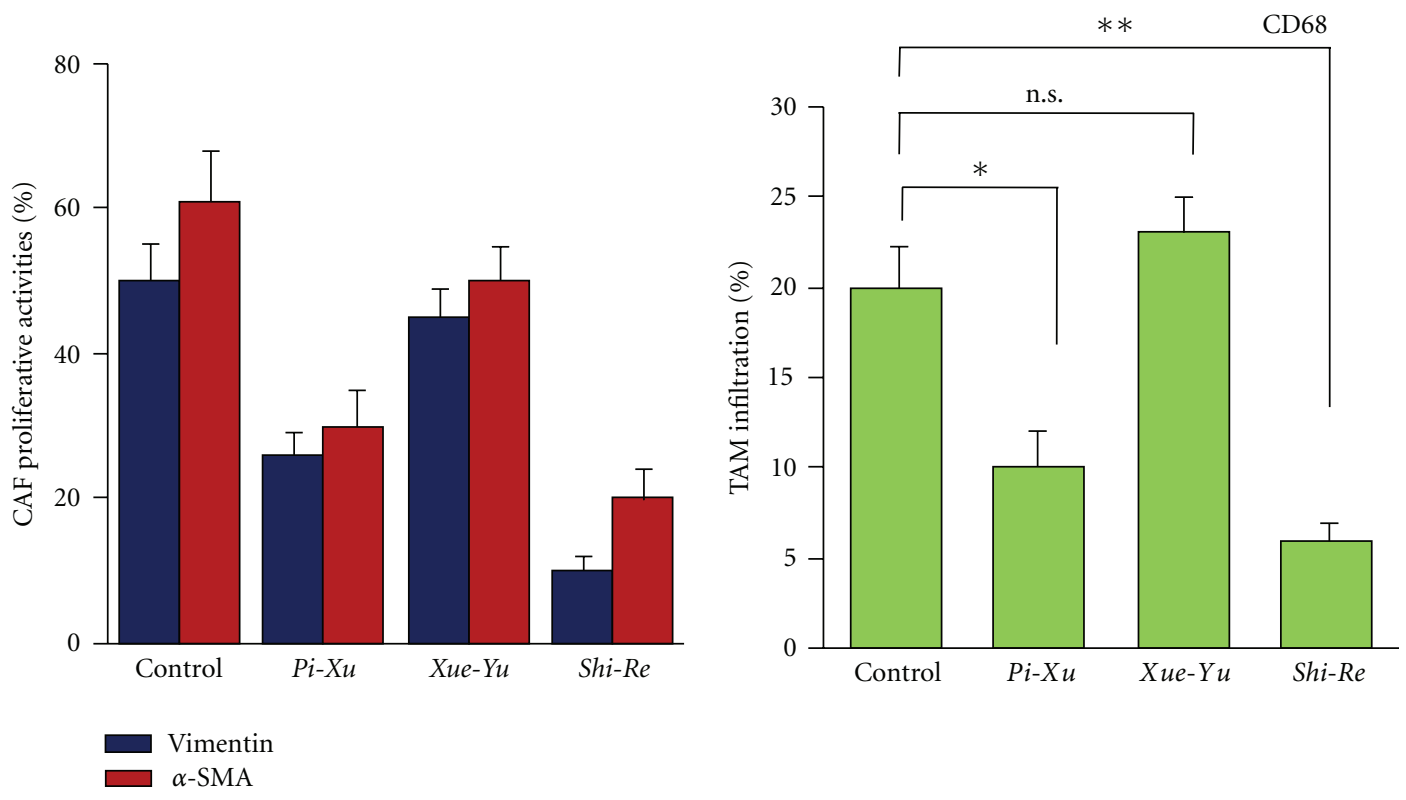

(b)

FIGURE 2: Alterations of tumor microenvironments under different ZHENG conditions. (a) Xenograft tumor models were established as described. On the next day, three types of ZHENG, namely, Shi-Re, Pi-Xu, and Xue-Yu were established. Tumors were obtained 4 wks after implantation. IHC staining for vimentin and $\alpha$-SMA on sections of tumors was performed for evaluating CAF proliferative activities (top). IHC staining for CD68 was performed for evaluating TAM infiltration (low). Original magnification, 200x. (b) CAF proliferative activity (left) and TAM infiltration (right) were quantitatively evaluated by calculating the ratio of vimentin or CD68 antibody positive staining area to the total area in each field, and the mean value from ten fields under $200 \mathrm{x}$ microscopy was indicated. ${ }^{*} P<0.05 ;{ }^{* *} P<0.01$; n.s.: not significant.

\subsection{Tumor Response under Different ZHENG Conditions to} Herbal Medicine Treatments. TCM usually means a comprehensive assessment of pathogenesis, location, and disease pathology, and the diagnosed ZHENG helps guide the application of Chinese herbal remedies. So, we used Huang lian jie $d u$ decoction (a traditional prescription used of treating Shi-Re ZHENG), Si jun zi decoction (a traditional prescription used of treating $P i-X u Z H E N G$ ), and Tao hong si $w u$ decoction (a traditional prescription used of treating Xue-Yu ZHENG) for Shi-Re, $P i-X u$, and Xue-Yu tumors, respectively. The herbal prescriptions used are shown in Table 1. We found that the herbal medicines had no or little effect on CAF proliferation or TAM infiltration (Figure 4(a)). We also evaluated the effects of the herbal medicines on SDF-1 secretion and CCL5 levels and found that the levels of which did not change after herbal medicine treatment (Figure 4(b)). Similarly, none of the herbal medicines had significant effects on CXCR4 or CCR5 expression in tumor cells (Figure 4(c)). These observations indicated that the herbal medicines had little effect on either the tumor or 


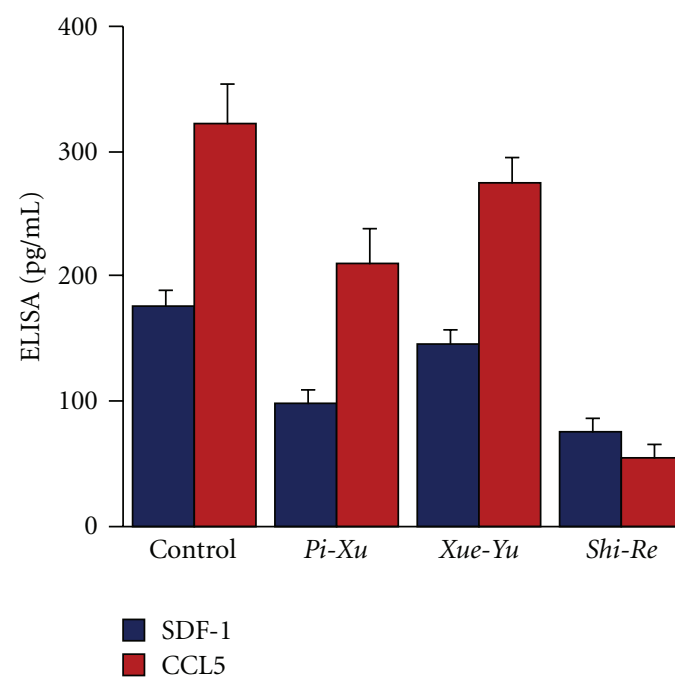

(a)

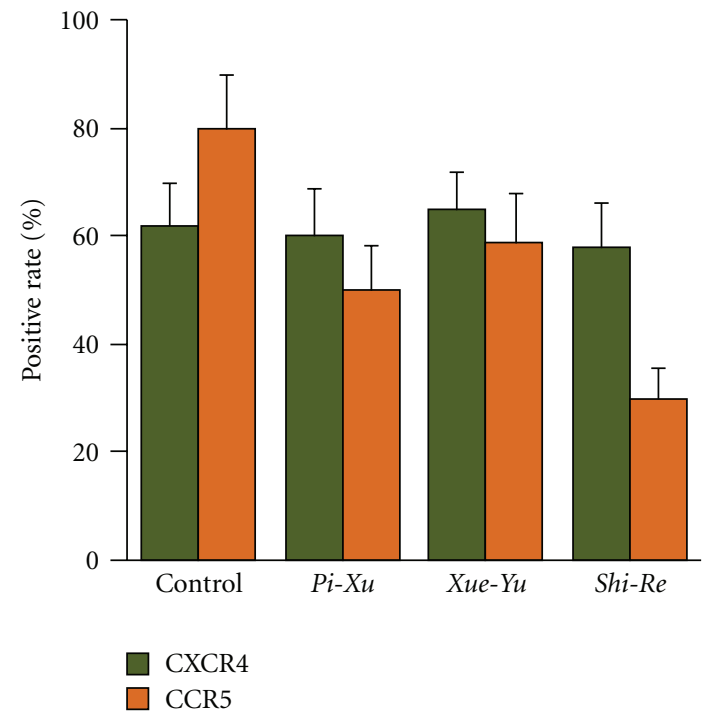

(c)

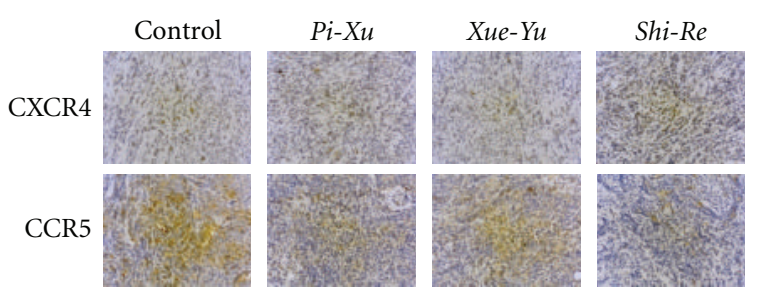

(b)

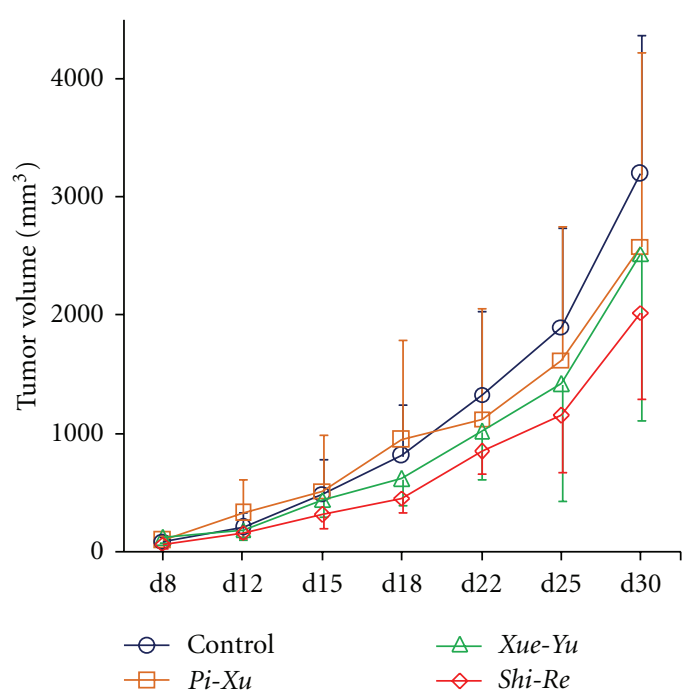

(d)

FIGURE 3: Correlation between microenvironment and tumor growth under different ZHENG conditions. (a) The levels of CAF- and TAMderived secreted cytokines, SDF-1 and CCL5, in tumor from the Shi-Re, Pi-Xu, and Xue-Yu ZHENG models were evaluated with ELISA assay. Data are expressed as the mean \pm SE. (b and c) IHC staining for CXCR4 and CCR5 on sections of tumors from ZHENG models was performed and representative photos are shown in (b). The positive rates of CXCR4 and CCR5 protein in tumors with indicated ZHENG are shown in (c). (d) Effect of ZHENG on tumor growth in a subcutaneously transplanted tumor model. Xenograft tumor model combined with ZHENG model was established as described in Figure 2(a). Mean volumes of tumors from each group were measured. Mean \pm standard deviation was determined for 10 mice in each group.

microenvironment. Finally we evaluated the effects of the herbal medicines on tumor growth and could not find any difference when the tumors were treated with different types of herbal medicine (Figure 4(d)). Therefore, our results suggest that a prescription based solely on ZHENG does not always result in a satisfactory response.

\subsection{Herbal-Medicine-Induced Alteration of Tumor Microen-} vironment Is Correlated with Treatment Response. Although tumors under different ZHENG conditions demonstrated differences in tumor microenvironment which may finally be reflected in tumor growth, the ZHENG conditions themselves seemed not to promote tumor growth. Thus, herbal medicines prescribed on the basis of the ZHENG condition alone did not affect tumor growth. To further understand the relatedness of tumor microenvironment, ZHENG, and the response to herbal treatment, we employed the Qingyihuaji formula $(\mathrm{QYHJ})$, a prescription based on TCM theory whereby pancreatic cancer is considered to be of Shi-Re origin. QYHJ has been used to treat pancreatic cancer for 

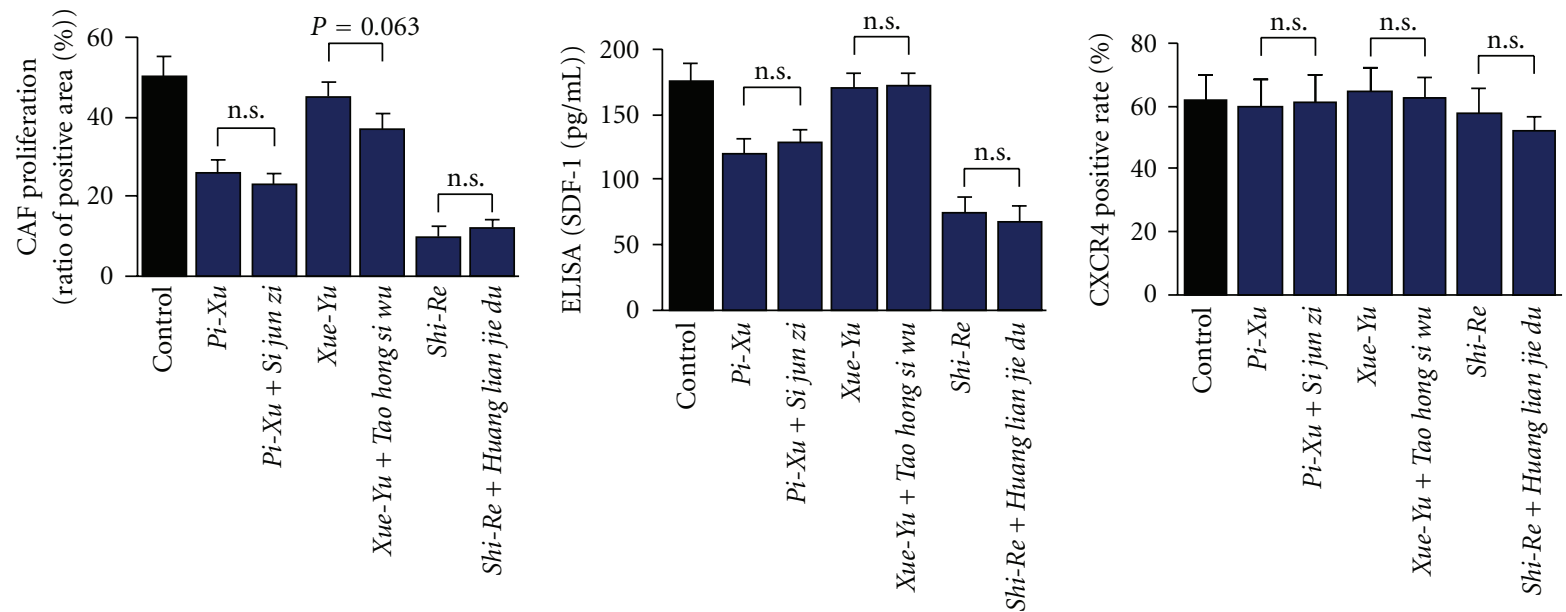

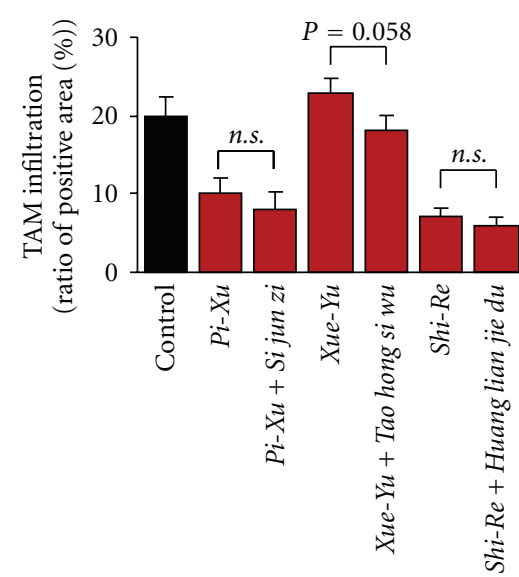

(a)

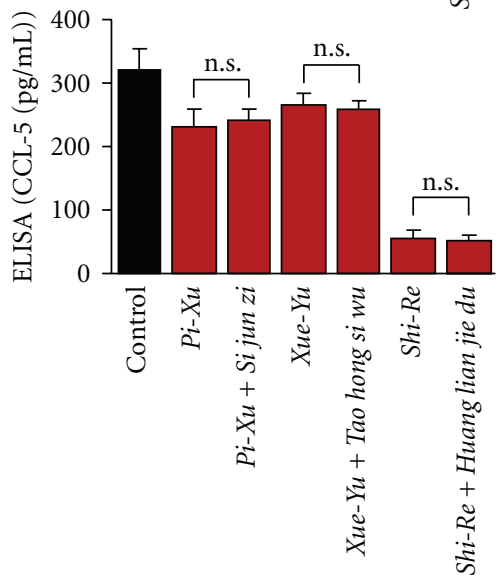

(b)

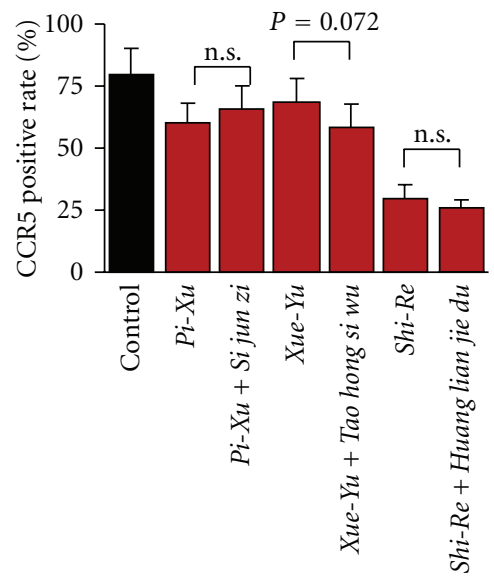

(c)

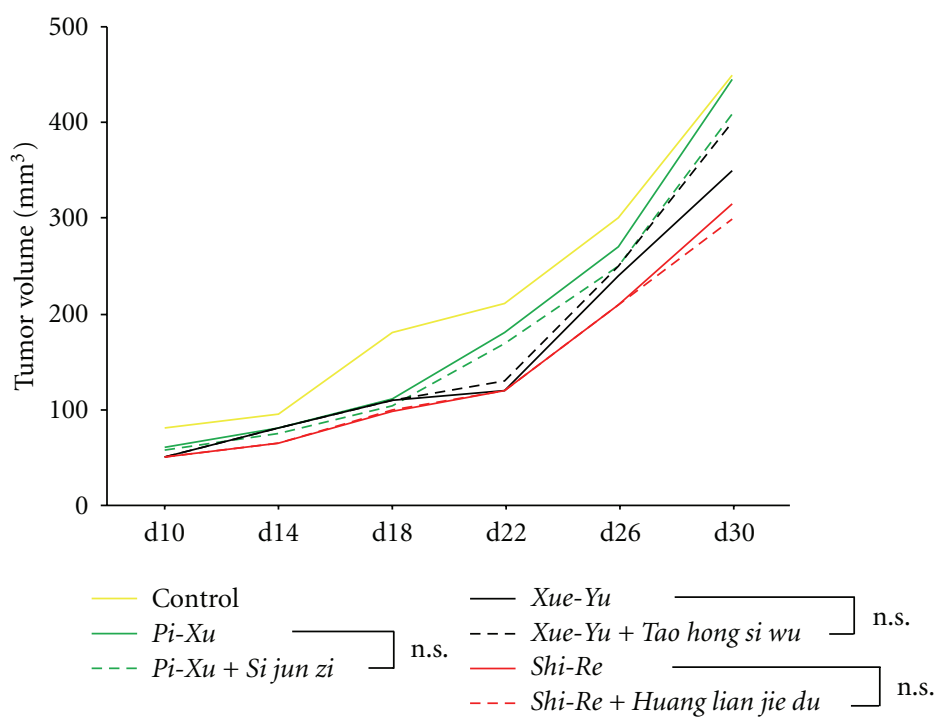

(d)

FIGURE 4: Response of tumors under different ZHENG conditions to herbal medicine treatments. (a) IHC staining for vimentin, $a$-SMA, and CD68 in tumors from the indicated group was performed for evaluating CAF proliferative activities and TAM infiltration, respectively. CAF proliferative activity and TAM infiltration were quantitatively evaluated as described in Figure 2(b). (b) The levels of SDF-1 and CCL5 in indicated tumors were evaluated with ELISA assay. Data are expressed as the mean \pm SE. (c) IHC staining for CXCR4 and CCR5 on sections of indicated tumors. The positive rates of CXCR4 and CCR5 protein in tumors with indicated ZHENG was calculated. (d) Effect of herbal medicine on subcutaneously transplanted tumor with different $Z H E N G$. The growth curves for each are shown. n.s.: not significant. 


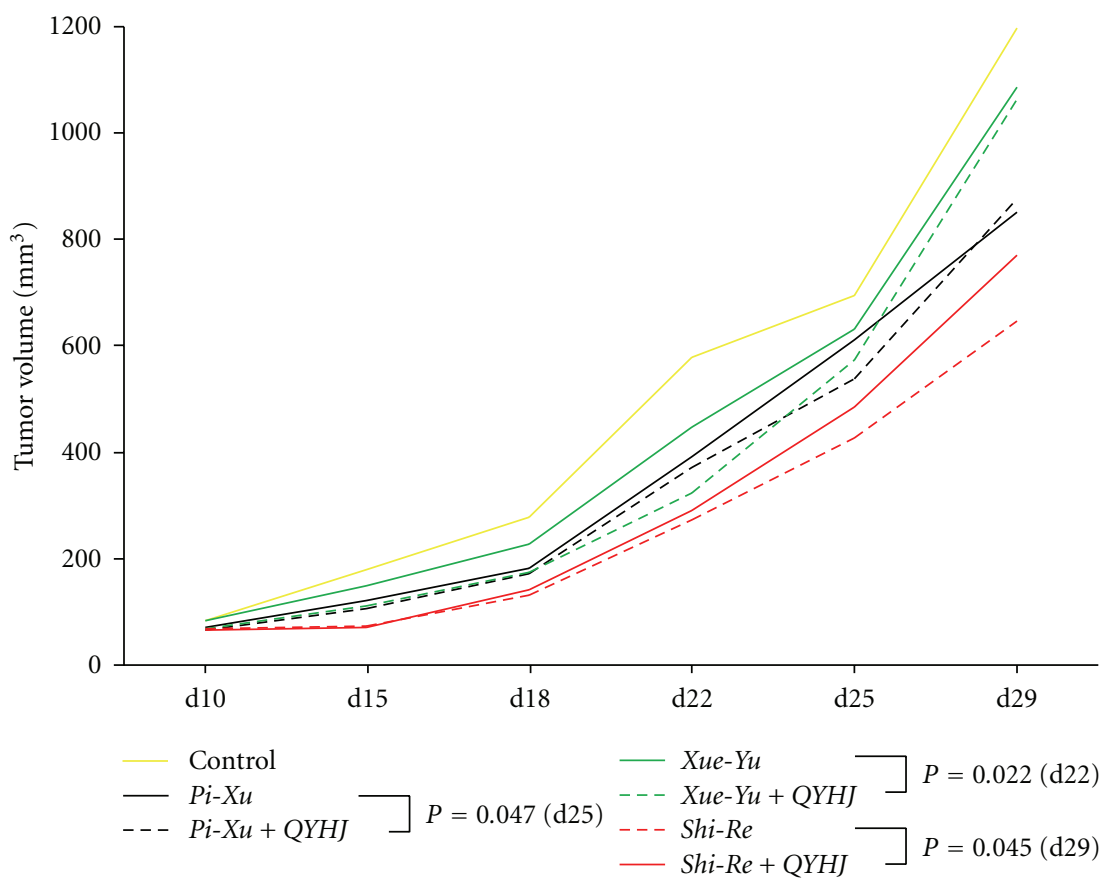

(a)

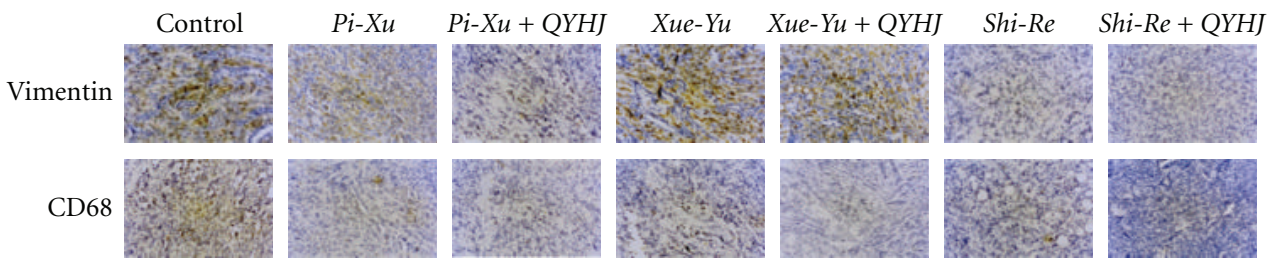

(b)
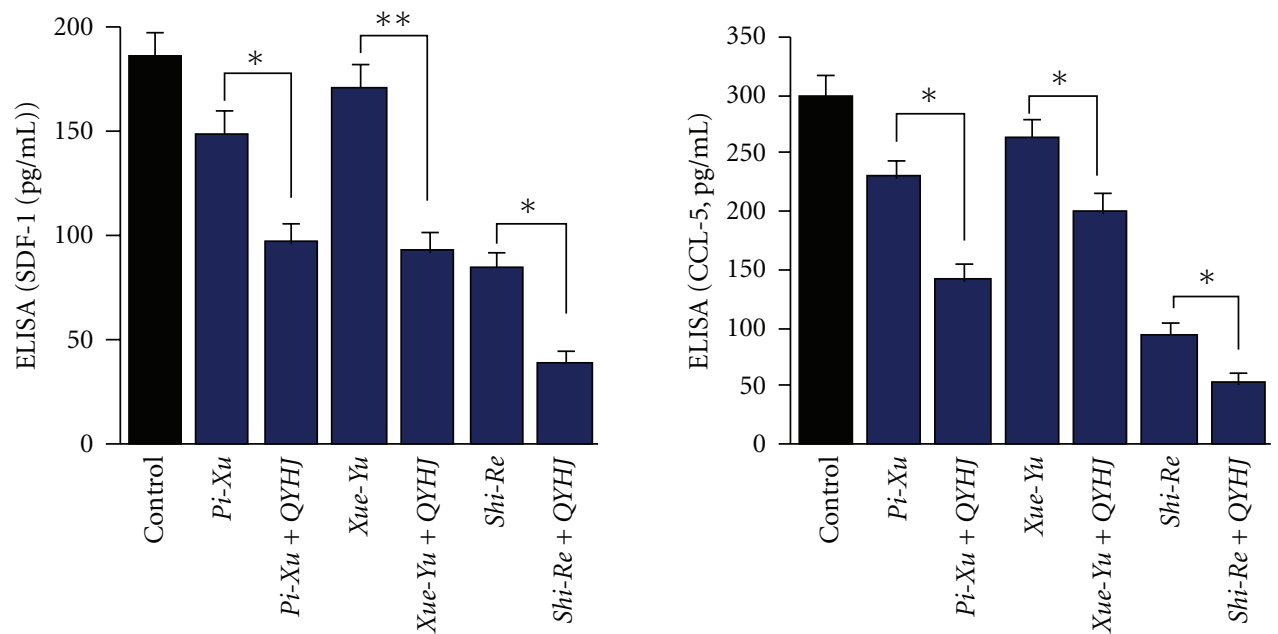

(c)

FIgURE 5: Treatment response to herbal medicine involved with modification of tumor microenvironment. (a) The antitumor effect of Qingyihuaji formula (QYHJ) on tumor with different ZHENG. (b) The effect of QYHJ on CAF proliferative activities and TAM infiltration were evaluated as described in Figure 2. (c) The effect of QYHJ on secreted SDF-1 and CCL5 levels were evaluated as described in Figure 3(a). ${ }^{*} P<0.05 ;{ }^{* *} P<0.01$. 
TABLE 1: Herbal prescriptions used in this study.

\begin{tabular}{ll}
\hline Prescription & Contents (g) \\
\hline Huang lian jie du decoction & Coptis root (9), baical skullcap root (6), amur corktree bark (6), cape jasmine fruit (9) \\
\hline Si jun zi decoction & Ginseng (9), largehead atractylodes rhizome (9), poria (9), liquorice root (6) \\
\hline Tao hong si wu decoction & $\begin{array}{l}\text { Peach seed (9), safflower (6), Chinese angelica root (9), rehmannia root (12), rhizome of szechwan lovage } \\
\text { (6), radix paeoniae rubra (9) }\end{array}$ \\
\hline Qingyihuaji formula & $\begin{array}{l}\text { Herba scutellariae barbatae (15), hedyotidis herba (15), amorphophallus konjac (15), coix seed (15), } \\
\text { fiveleaf gynostemma herb (15), java amomum fruit (10) }\end{array}$ \\
\hline
\end{tabular}

many years $[15,16]$. We first established mouse tumor models with the accompanying ZHENG conditions Shi-Re, $P i-X u$, and $X u e-Y u$. The mice were then treated with QYHJ and the tumor microenvironment and tumor growth were evaluated. We found that tumors of the different ZHENG condition models exhibited altered tumor microenvironments (Figures 5(b) and 5(c)), which is consistent with our previous observations (Figure 2). The QYHJ treatments altered the tumor microenvironments in the Shi-Re, $P i-X u$, Xue-Yu models dramatically, as demonstrated by decreased CAF proliferation and TAM infiltration (Figure 5(b) and $5(c))$. Then when we correlated tumor microenvironment alteration with tumor growth, we surprisingly found that QYHJ treatment led to growth inhibition of tumors under different $Z$ HENG conditions, although the inhibition rate varied among the different ZHENG (Figure 5(a)). This suggests that disease identification is sometime a requisite for the treatment of cancer with TCM. Taken together, these results indicate that a combination of disease diagnosis and $Z$ HENG identification is essential for clinical TCM practice in cancer treatment. They also showed a relatedness between the tumor environment and $Z H E N G$, and treatment response to herbal medicine involved the modification of the tumor microenvironment.

\section{Discussion}

In this study, we established three different ZHENG mouse models according to TCM theory. We identified alterations in the tumor microenvironment under different $Z H E N G$ conditions. These tumor microenvironment modifications mediated a correlation between the ZHENG condition and response to herbal treatment. Therefore our study revealed a molecular basis for ZHENG in pancreatic cancer.

In TCM clinical practice, $Z H E N G$ helps guide the remedy prescription and therefore has an important position in the TCM system, that is, ZHENG is the key to recognizing diseases and the foundation to treat them. However, because of the complexity of the concepts (e.g., a single $Z H E N G$ involves multiple anatomical systems) and lack of nonprofessional descriptions, research of $Z H E N G$ is difficult to advance. The molecular basis underlying ZHENG in TCM remains unclear.

It has been confirmed that tumor cells do not act in isolation, but rather subsist in a rich microenvironment provided by resident fibroblasts, inflammatory cells, endothelial cells, pericytes, leukocytes, and extracellular matrix [17]. It is increasingly appreciated that, as the cancer progresses, the surrounding microenvironment is activated in support, coevolving through continuous paracrine communication and supporting carcinogenesis [3]. Pancreatic ductal adenocarcinoma is characterized by an extensive stromal response called desmoplasia. Within the tumor stroma, CAFs are the primary cell type; the importance of the role of CAFs in tumor progression is now well accepted. CAFs produce large amounts of secreted factors, including CXC, CC chemokines, and other inflammatory mediators that promote the proliferation, invasion, and metastasis of cancer cells [18]. It is also accepted that large numbers of tumor-associated leukocytes infiltrate solid tumors, and TAMs represent a major and important component of these leucocytes, which are driven toward functions that support cancer progression and poorer prognosis [12]. Therefore the stromal elements of tumors hold prognostic, as well as response-predictive, information. Abundant targeting opportunities within the tumor microenvironment are continually identified [3].

As TCM sustains systematic theories and is a holistic approach to health, and our previous study has indicated a correlation between $Z$ HENG and levels of cytokines related to CAF and TAM [4], we hypothesized a correlation between the tumor microenvironment and the ZHENG syndromes of TCM. Thus we evaluated the tumor microenvironment by immunostaining for CAF and TAM and surprisingly found differences in microenvironment alterations under different ZHENG conditions. Furthermore, the alterations in CAF proliferative activity and TAM infiltration led to changed levels of CAF- and TAM-derived secreted cytokines which finally affected tumor growth.

Based on TCM theory and clinical experience, patients with pancreatic cancer usually exhibit Shi-Re, $P i-X u$, or $X u e-Y u$, and respective herbal decoctions for removing heat and dampness (Huang lian jie $d u$ ), reinforcing Qi and strengthening the spleen (Si jun zi), and promoting blood circulation and removing blood stasis (Tao hong si wu) are always prescribed. However the efficacy of these remedies is not always satisfactory. In the current study, the application of decoctions of these herbal medicines had little effect on tumor growth or the tumor microenvironment. This observation seems to contradict the TCM theories of treating the same disease with different methods and treating different diseases with the same methods. There are many reasons that may account for the lack of response to the TCM treatments in the present study. One is that each prescription 
has its priority and focus, although they are within the same category for the same ZHENG. Another reason is that apart from the traditional relationship between disease and ZHENG, there may also exist analogous ZHENG in the same disease, which means that different patients who suffer from the same disease manifest the common basic ZHENG in spite of slight differences in their accompanying symptoms. Therefore, we can use a basic prescription with slight modifications to treat accompanying symptoms. In fact recent research emphasized the principle of analogous ZHENG existing in the same disease in TCM clinical practice [19], especially in cancer treatment. These two reasons may partially explain why the prescriptions based on ZHENG used in the present study had little effect on tumor growth.

The integration of disease diagnosis and identification of ZHENG have been widely used in cancer treatment [20]. Based on our previous studies, pancreatic cancer is characterized by dampness, heat, and toxicity and should be treated by removing heat and dampness, detoxification and resolving a mass [21]. According to this recognition, we recommend the QYHJ formula in the treatment of pancreatic cancer. The results of our clinical studies suggest that treatment with QYHJ resulted in prolonged survival time for patients with pancreatic cancer $[15,21]$. Animal studies showed that QYHJ could inhibit the growth of subcutaneously transplanted pancreatic tumors in nude mice [16]. Just as we can see from this study, QYHJ had an effect on tumor growth and the tumor microenvironment, although the effect varied depending on the ZHENG type. Therefore, our study suggests an intrinsic disease-specific ZHENG, which should be considered during TCM practice. The study also indicated that the tumor microenvironment influences the tumor response to herbal medicine treatment.

In conclusion, our study showed alterations in the tumor microenvironment under different ZHENG conditions. We also confirmed a relatedness between the tumor environment and ZHENG, and herbal medicine treatments modified the tumor microenvironment. This study partially unveiled the molecular basis of TCM ZHENG in pancreatic cancer.

\section{Acknowledgments}

This study was supported by National Science Foundation of China (81001061); Shanghai Nature Science Fund, Shanghai, China (09ZR1406800); Doctoral Programs Foundation of Ministry of Education of China (20090071120076); Shanghai Science and Technology Committee Rising-Star Program (11QA1401300); Medical Talents Training Program of Health Bureau of Shanghai (XYQ2011008); Shanghai Municipal Health Bureau Grant (KY148).

\section{References}

[1] A. P. Lu, X. R. Ding, and K. J. Chen, "Current situation and progress in integrative medicine in China," Chinese Journal of Integrative Medicine, vol. 14, no. 3, pp. 234-240, 2008.

[2] D. Hanahan and R. A. Weinberg, "Hallmarks of cancer: the next generation," Cell, vol. 144, no. 5, pp. 646-674, 2011.
[3] K. Pietras and A. Östman, "Hallmarks of cancer: interactions with the tumor stroma," Experimental Cell Research, vol. 316, no. 8, pp. 1324-1331, 2010.

[4] H. Y. Dai, Peng Wang, L. Y. Feng et al., "The molecular mechanisms of traditional Chinese medicine ZHENG syndromes on pancreatic tumor growth," Integrative Cancer Therapies, vol. 9, no. 3, pp. 291-297, 2010.

[5] Z. Chen, L.-M. Liu, and Y.-B. Liu, "Characteristics of W256 tumor growth and liver metastasis in mice model with Blood stasis syndrome," Zhong Yi Yao Xue Kan, vol. 21, no. 6, pp. 866867, 2003.

[6] J. A. Joyce and J. W. Pollard, "Microenvironmental regulation of metastasis," Nature Reviews Cancer, vol. 9, no. 4, pp. 239252, 2009.

[7] K. Polyak, I. Haviv, and I. G. Campbell, "Co-evolution of tumor cells and their microenvironment," Trends in Genetics, vol. 25, no. 1, pp. 30-38, 2009.

[8] A. Neesse, P. Michl, K. K. Frese et al., "Stromal biology and therapy in pancreatic cancer," Gut, vol. 60, no. 6, pp. 861-868, 2011.

[9] L. A. Kunz-Schughart and R. Knuechel, "Tumor-associated fibroblasts (Part I): active stromal participants in tumor development and progression?" Histology and Histopathology, vol. 17, no. 2, pp. 599-621, 2002.

[10] A. Mantovani and A. Sica, "Macrophages, innate immunity and cancer: balance, tolerance, and diversity," Current Opinion in Immunology, vol. 22, no. 2, pp. 231-237, 2010.

[11] C. Steidl, T. Lee, S. P. Shah et al., "Tumor-associated macrophages and survival in classic Hodgkin's lymphoma," The New England Journal of Medicine, vol. 362, no. 10, pp. 875-885, 2010.

[12] A. Mantovani, T. Schioppa, C. Porta, P. Allavena, and A. Sica, "Role of tumor-associated macrophages in tumor progression and invasion," Cancer and Metastasis Reviews, vol. 25, no. 3, pp. 315-322, 2006.

[13] N. A. Bhowmick, E. G. Neilson, and H. L. Moses, "Stromal fibroblasts in cancer initiation and progression," Nature, vol. 432, no. 7015, pp. 332-337, 2004.

[14] F. Marchesi, P. Monti, B. E. Leone et al., "Increased survival, proliferation, and migration in metastatic human pancreatic tumor cells expressing functional CXCR4," Cancer Research, vol. 64, no. 22, pp. 8420-8427, 2004.

[15] H. Ouyang, P. Wang, Z. Meng et al., "Multimodality treatment of pancreatic cancer with liver metastases using chemotherapy, radiation therapy, and/or Chinese herbal medicine," Pancreas, vol. 40, no. 1, pp. 120-125, 2011.

[16] P. Wang, Z. Chen, Z. Q. Meng et al., "Ski acts as therapeutic target of Qingyihuaji formula in the treatment of SW1990 pancreatic cancer," Integrative Cancer Therapies, vol. 9, no. 1, pp. 50-58, 2010.

[17] R. R. Langley and I. J. Fidler, "The seed and soil hypothesis revisited-The role of tumor-stroma interactions in metastasis to different organs," International Journal of Cancer, vol. 128, no. 11, pp. 2527-2535, 2011.

[18] D. Mahadevan and D. D. Von Hoff, "Tumor-stroma interactions in pancreatic ductal adenocarcinoma," Molecular Cancer Therapeutics, vol. 6, no. 4, pp. 1186-1197, 2007.

[19] W. J. Wang, "Pay more attention to "Analogous syndrome complex in the same disease and treating the same disease with analogous methods"', Zhong Xi Yi Jie He Xue Bao, vol. 6, no. 5, pp. 441-445, 2008. 
[20] L. M. Liu, "The prospective of integrative medical treatment of oncology in the next ten years," Zhongguo Zhong Xi Yi Jie He Za Zhi, vol. 31, no. 7, pp. 870-872, 2011.

[21] Y.-H. Shen, L.-M. Liu, Z. Chen et al., "Combined with chemotherapy for treatment of 32 cases of advanced pancreatic cancer," Journal of Traditional Chinese Medicine, vol. 47, no. 2, pp. 115-117, 2006. 


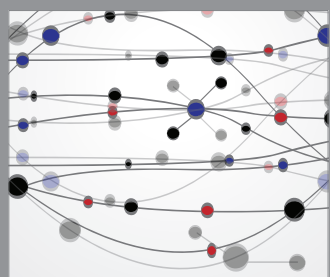

The Scientific World Journal
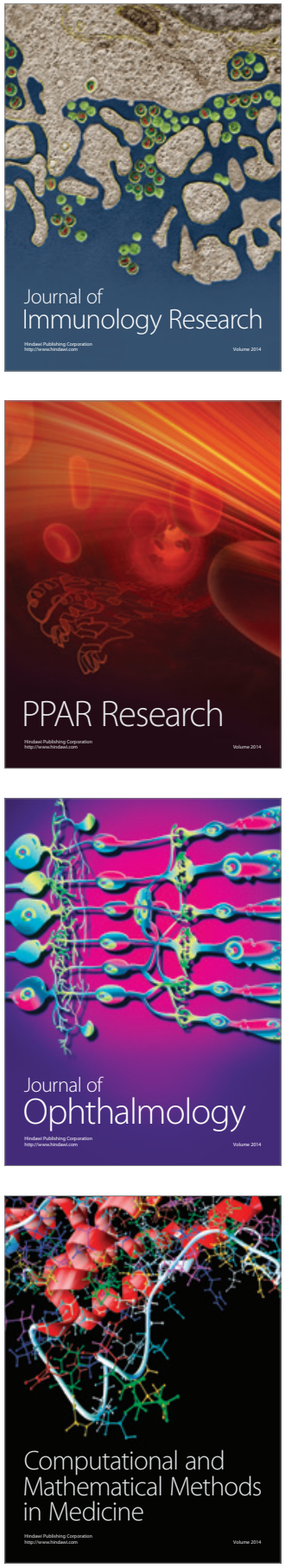

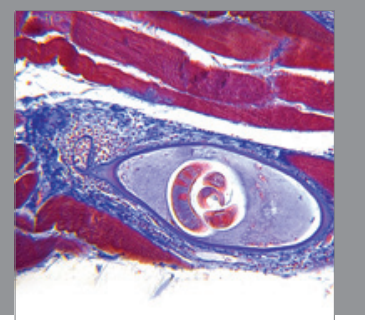

Gastroenterology

Research and Practice
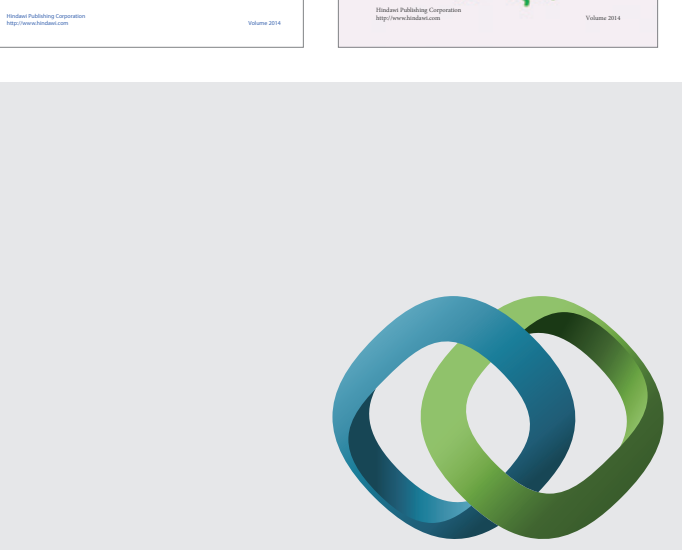

\section{Hindawi}

Submit your manuscripts at

http://www.hindawi.com
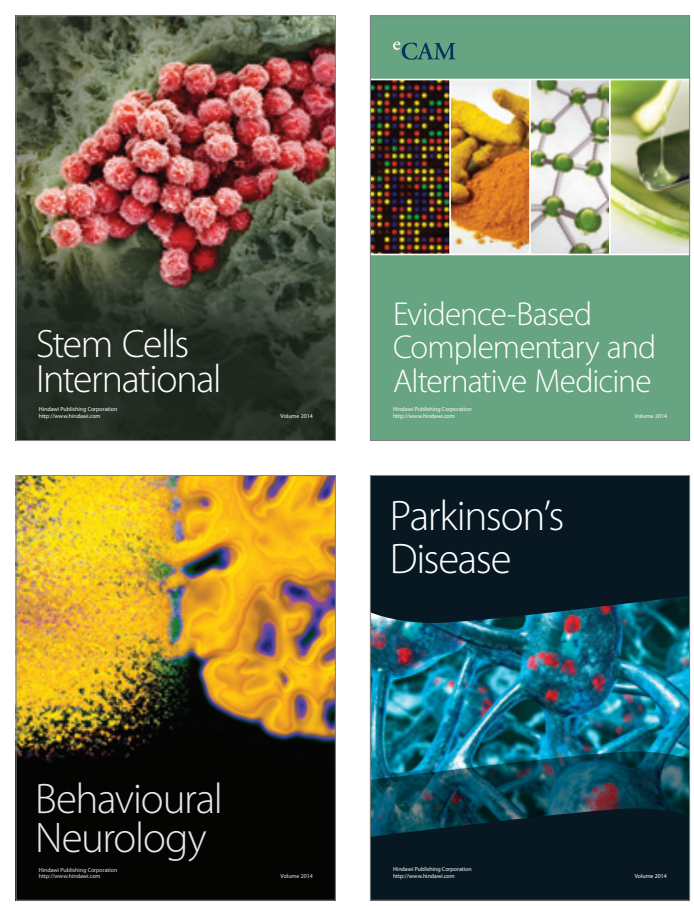

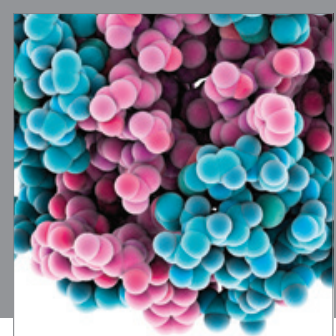

Journal of
Diabetes Research

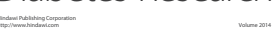

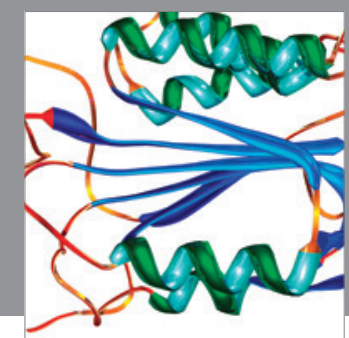

Disease Markers
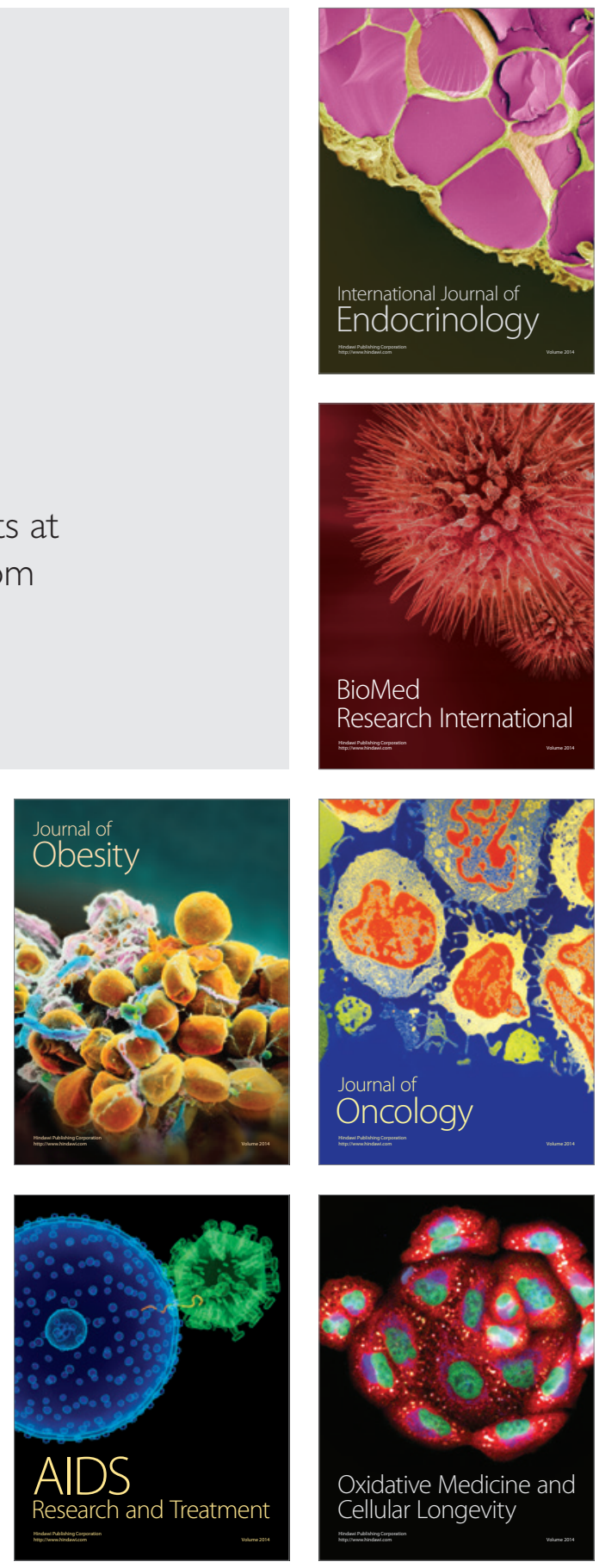